\title{
Motivación autodeterminada en deportistas colombianos con discapacidad visual ${ }^{*}$
}

\author{
Self-determined motivation in Colombian athletes \\ with visual impairment
}

Motivação auto-determinada em atletas colombianos
com deficiência visual

Ángela María Urrea*

Félix Guillén

Recibido 28. 02. 2016 • Arbitrado 26. 03. 2016 • Aprobado 13. 04. 2016

\section{Resumen}

El propósito de este estudio ha sido investigar la relación existente entre la motivación con el género, la edad y el tipo de deficiencia en deportistas colombianos en situación de discapacidad visual. Los participantes en este estudio fueron 109 sujetos con edades entre 18 y 35 años $(M=26.05 ; D T=5.41)$. Se administró un cuestionario sociodemográfico que recogía información acerca del género, la edad, el deporte practicado, el tipo de discapacidad y la causa de la discapacidad, asimismo se utilizó la Escala de Motivación Deportiva (SMS-28). Los resultados revelan diferencias estadísticamente significativas en función del géne-

* Articulo presenta resultados de la investigación denominada "Fortaleza mental en deportistas de alto rendimiento con deficiencia visual", realizada para optar el título de doctor en psicología deportiva de la Universidad de las Palmas de Gran Canarias, España, 2010-2015.

* PhD. en Comportamiento Humano en Contextos Deportivos, de Ejercicio y de Actividad Física, Universidad de las Palmas de Gran Canaria; Mg. en Psicología de la Actividad Física y del Deporte, Universidad Autónoma de Madrid; Psicóloga, Universidad de San Buenaventura (Cali). Docente Tiempo Completo Ocasional, Institución Universitaria de Envigado, Facultad de Ciencias Sociales, Envigado, Antioquia, Colombia. Coordinadora Semillero de investigación en Psicología de la Actividad Física y del Deporte, angela.urrea@psicologiadeldeporte.com.co

*** PhD. en Psicología. Universidad de la Laguna. Licenciado en Filosofía y Ciencias de la Educación (Sección Psicología), Universidad de La Laguna. Docente Tiempo Completo, Universidad de Las Palmas de Gran Canaria, Facultad de Ciencias de la Actividad Física y el Deporte, Departamento de Psicología y Sociología. Las Palmas de Gran Canaria, España, felix.guillen@ulpgc.es 
ro en las subescalas de motivación extrínseca, específicamente, en regulación introyectada y externa. En cuanto al análisis de correlaciones se pone de manifiesto, que los deportistas tienen una relación significativa y positiva entre la Motivación Extrínseca (ME) y la Motivación Intrínseca (MI) en cada una de sus subescalas, siendo la más fuerte, la relacionada con la Regulación Identificada (RI) y la Motivación Intrínseca al Logro (MIL); mientras que la más baja es la Regulación Externa (RE) con la Motivación Intrínseca a la Experiencia (MIE), cumpliendo así con lo propuesto por Deci y Ryan (1985). Por último, los datos indican que la Amotivación (A) es significativamente baja.

Palabras clave: motivación, tipo de discapacidad, situación de discapacidad visual, autodeterminación y deporte.

\section{Abstract}

The purpose of this research has been to investigate the existent relation between motivation and the gender, age and type of deficiency in Colombian sportsmen in condition of visual disabilities. The participants in this study were 109 subjects in age from 18 to 35 years old. $(M=26.05$; DT $=5.41)$. It was dispensed a socio-demographic questionary that collected information about gender, age, sport practiced, type of disability and cause of disability, likewise, the Sport Motivation Scale (SMS-28) was used. The results reveal differences statistically significant in function of gender and subscales of intrinsic motivation, specifically, in introjected and external regulation. As to the correlation analysis it shows that the sportsmen have a significant and positive relation between the Extrinsic Motivation (EM) and Intrinsic Motivation (IM) in each one of its scales, being the strongest the one related with Identified Regulation (IR) and Intrinsic Motivation to Accomplish (IMA); while the lowest is the External Regulation (ER) with the Instrinsic Motivation to the Experience (IME), thus fulfilling what was proposed by Deci and Ryan (1985). By last, data shows that Amotivation (A) is significatively low. 
Key words: motivation, type of disability, visual disability situation, self-determination and sport.

\section{Resumo}

O objetivo deste estudo foi investigar a relação entre motivação para sexo, idade e tipo de deficiência em atletas colombianos em situações de deficiência visual. Os participantes neste estudo foram 109 indivíduos com idades entre 18 e 35 anos $(M=26.05, \mathrm{SD}=5,41)$. um questionário sócio-demográfico coletadas informações sobre sexo, idade, praticava esporte, o tipo de deficiência e a causa da deficiência foi dado igualmente Desporto Escala de Motivação (SMS-28) foi utilizado. Os resultados mostram diferenças estatisticamente significativas por sexo em subescalas motivação extrínseca, especificamente na regulação introjetada e externa. Quanto à análise de correlação é evidente que os atletas têm uma relação significativa e positiva entre a motivação extrínseca (ME) e motivação intrínseca (MI) em cada uma das sub-escalas, sendo o mais forte, o relacionado regulação identificada (RI) e Achievement motivação intrínseca (MIL); enquanto o mais baixo é a regulação externa (RE) com motivação intrínseca para Experience (MIE), assim reunião proposta pela Deci e Ryan (1985). Finalmente, os dados indicam que o amotivação (A) é significativamente baixa.

Palavras-chave: motivação, deficiência, condição de deficiência visual, auto-determinação e do desporto.

\section{Introducción}

La motivación es una de las variables más estudiadas en el área de la Psicología del Deporte, se ha investigado en los diferentes agentes que componen el deporte, entre ellos, deportistas, árbitros y jueces deportivos, padres de familia, entrenadores, etc. Son múltiples y muy diversos los factores que pueden determinar la motivación y los que la pueden dificultar. Es de tener en cuenta, que esta variable es un componente 
fundamental para la práctica del deporte, actividad física o recreación, es la que ayuda a determinar la elección, intensidad, persistencia, rendimiento y adherencia a un programa específico y/o permite la optimización para alcanzar altos niveles de rendimiento. Es por ello, que en este estudio se ha seleccionado la motivación autodeterminada para dar cuenta cómo se comportan los deportistas con discapacidad visual.

La motivación puede definirse como la dirección e intensidad del esfuerzo (Weinberg y Gould, 2010). La dirección del esfuerzo se refiere a si el sujeto busca, se aproxima o se siente atraído por ciertas situaciones, por ejemplo: un deportistas lesionado, para conseguir un tratamiento médico adecuado; mientras que la intensidad de esfuerzo se refiere a la cantidad de empeño que un sujeto emplea en una situación determinada, por ejemplo: cuando un levantador de pesas puede entrenarse cuatro días a la semana igual que sus amigos, pero diferenciarse de ello en la intensidad o el enorme esfuerzo que realiza en cada sesión de ejercicios.

Por su parte, Cox (2009, p. 121) señala que

La motivación proviene del interior es intrínseca por naturaleza. Las tareas que son intrínsecamente motivantes son las que el deportista realiza debido a que le resultan interesantes. La volición y el control personal son componentes fundamentales de la motivación interna. Un deportista se compromete de forma espontánea con una actividad que le resulta intrínsecamente motivante. En este caso no son necesarias las recompensas ni ningún tipo de control externo. Un deportista habilidoso que se muestra muy motivado solamente por motivos externos es un peón eficaz. La conducta será regulada por factores externos.

Siguiendo con los motivos internos y externos, se retoma a Bakker, Whiting y Van der Brug (1993), los cuales relacionan la motivación (combinación de factores internos (personales) y externos (de situación) con el estado del organismo al que se considera responsable de la realización de una determinada actividad en un punto preciso del tiempo. 


\section{Teoría de la autodeterminación}

La TAD es una macro-teoría de la motivación humana que tiene relación con el desarrollo y funcionamiento de la personalidad dentro de los contextos sociales. La teoría analiza el grado en que las conductas humanas son volitivas o autodeterminadas, es decir, el grado en que las personas realizan sus acciones al nivel más alto de reflexión y se comprometen en las acciones con un sentido de elección (Deci \& Ryan, 2012). Esto es, el grado en que las personas realizan sus acciones de forma voluntaria, por propia elección (Carratalá, 2004). Se basa en una meta-teoría organísmico-dialéctica que asume que las personas son organismos activos, con tendencias innatas hacia el crecimiento psicológico y del desarrollo, esforzándose por dominar los desafíos continuados e integrar sus experiencias de forma coherente con su voluntad.

Deci \& Ryan (2012) introdujeron el continuum de la motivación para detallar las diferentes formas de motivación extrínseca y los factores contextuales que promueven o impiden la interiorización e integración de éstos en la regulación de las conductas (Deci \& Ryan, 2000). Estos autores establecen una taxonomía donde la motivación se estructura en forma de un continuo que abarca los diferentes grados de autodeterminación de la conducta. Este continúo de la motivación abarca desde la conducta no autodeterminada, hasta la conducta autodeterminada. El recorrido de un tipo de conducta a otra abarca tres tipos fundamentales de motivación: la amotivación, la motivación extrínseca y la motivación intrínseca. A su vez, cada uno de estos tipos de motivación tiene su propia estructura y está regulada por el sujeto de forma interna o externa.

Para completar el modelo, cada uno de los tipos de motivación está determinado por una serie de procesos reguladores, como pueden ser valores, recompensas, autocontrol, intereses, diversión, satisfacción, etc. A continuación, se describen los diferentes niveles de motivación propuestos por Deci \& Ryan (2012, 2002, 2000).

La amotivación, se encuentra en el extremo del continuo de auto-determinación, esta corresponde a la falta absoluta de motivación, tanto 
intrínseca como extrínseca, es decir, es el grado más bajo de autodeterminación (García-Calvo, 2004). Esta hace referencia a la falta de intencionalidad para actuar (Deci \& Ryan, 2012; Edmunds, Ntoumanis \& Duda, 2006) y se produce cuando el individuo no valora una actividad (Deci \& Ryan, 2008), no sintiéndose competente para hacerla (Bandura, 2001), o no esperando la consecución del resultado esperado (Seligman, 1975). Los atletas que están desmotivados practican sin propósito, experimentan efectos negativos, como apatía, incompetencia, depresión, y no buscan objetivos afectivos, sociales ni materiales (Vallerand, 2001). En este sentido, Villacorta, Koestner \& Lekes (2003), consideran cuatro tipos dentro de la amotivación: a) una amotivación relacionada con las creencias de capacidad/habilidad (por lo que la amotivación es el resultado de la falta de habilidad para realizar una conducta); b) un segundo tipo, que es resultado de las creencias del individuo (que piensa que la estrategia a seguir no dará el resultado esperado); c) otra tercera variante está relacionada con las creencias de capacidad y esfuerzo (la conducta requiere demasiado esfuerzo, y el individuo no quiere implicarse en ese esfuerzo), d) y un cuarto tipo de amotivación, relacionado con las creencias de impotencia, donde el individuo percibe que el esfuerzo no tendrá gran trascendencia, teniendo en cuenta la gran tarea que debe realizar.

Por su parte, la motivación extrínseca, está determinada por recompensas o agentes externos. Dentro de la teoría de la Integración Organísmica, la investigación ha determinado cuatro tipos de motivación extrínseca (Deci \& Ryan, 2000, 2002, 2008, 2012): regulación externa, regulación introyectada, regulación identificada y regulación integrada.

El primer tipo de motivación extrínseca es la regulación externa. Es la forma menos autodeterminada de motivación extrínseca y, por tanto, la de mayor contraste con la motivación intrínseca. La conducta se realiza para satisfacer una demanda externa o por la existencia de premios o recompensas. Los individuos experimentan la conducta sintiéndose controlados o alienados (Deci \& Ryan, 2000). La regulación externa constituye el típico caso de motivación por la búsqueda de 
recompensas o evitación de castigos (Deci \& Ryan, 2000). Esta forma de regulación se caracteriza por tener un locus de control externo. Así, sería la situación en que se encuentran muchos individuos que practican actividad física porque "los demás me dicen que debo hacerlo", "los amigos, la familia, mi pareja, etcéra me presionan para que haga ejercicio", "el entrenador me dice que debo mejorar mi marca en 5 segundos si quiero hacer algo esta temporada", etc.

El segundo tipo de motivación extrínseca es la regulación introyectada, también llamada introyección o regulación auto-ejecutada. Este tipo de regulación está asociada a las expectativas de auto-aprobación, evitación de la ansiedad y el logro de mejoras del ego, en aspectos tales como el orgullo (Deci \& Ryan, 2000). La regulación de la conducta sigue teniendo todavía un locus de control externo. Los motivos de participación en una actividad son principalmente el reconocimiento social, las presiones internas o los sentimientos de culpa (García-Calvo, 2004). El individuo ejerce presión sobre sí mismo para regular su comportamiento. En este estadio encontraríamos a aquellas personas que muestran opiniones o sensaciones respecto a la práctica como "me siento mal si no he practicado", "siento que he fallado si no he realizado un poco de ejercicio", o "me siento mal por haber faltado al entrenamiento".

El tercer nivel de regulación dentro del continuo de la motivación propuesto por Deci \& Ryan (2002) es la de regulación identificada. La conducta es altamente valorada y el individuo la juzga como importante, por lo que la realizará libremente, aunque la actividad no sea agradable (Carratalá, 2004). Cuando una persona se identifica con la acción o el valor que expresa, de forma consciente, existe un alto grado de autonomía percibida (Deci \& Ryan, 2000; 2002). Por ejemplo, imaginemos a un sujeto que sale a correr desde hace poco tiempo. Es posible que la actividad no le resulte agradable, pero sabe que le reportará beneficios físicos y psicológicos. También en el ámbito competitivo podemos encontrar ejemplos de este tipo. Probablemente el atleta saltador de pértiga no encuentre demasiado agradable el trabajo aeróbico de principio de temporada, pero sabe que le ayudará a mejorar su 
capacidad de recuperación entre esfuerzos y le permitirá crear la base sobre la que asimilar entrenamientos más intensos.

El último nivel de regulación es el de la regulación integrada. En este caso, la conducta se realiza libremente. La integración ocurre cuando la persona evalúa la conducta y actúa en congruencia con sus valores y sus necesidades. Sería el caso de aquellas personas que se comprometen con la práctica físico-deportiva porque forma parte de su estilo de vida activo.

$\mathrm{Y}$, por último, se define la motivación intrínseca, como aquella relacionada con la necesidad de explorar el entorno, la curiosidad y el placer que se experimenta al realizar una actividad, sin recibir una gratificación externa directa. El desarrollo de la actividad en sí constituye el objetivo y la gratificación, suscitando también sensaciones de competencia y autorrealización. Un aspecto importante de la conducta intrínsecamente motivada es que el interés por la actividad y las necesidades de competencia y autorrealización subsisten incluso después de haberse alcanzado la meta. Autores como Vallerand, Blais, Brière \& Pelletier (1989) y Vallerand, Pelletier, Blais, Brière, Senécal \& Vallières (1993), plantean que existen tres tipos de motivación intrínseca: motivación intrínseca hacia el conocimiento (el sujeto se compromete en una actividad por el placer y la satisfacción que experimenta mientras intenta aprender), motivación intrínseca hacia la ejecución (el sujeto se compromete en la actividad por placer mientras intenta mejorar o superarse a sí mismo), y la motivación intrínseca hacia la estimulación (el sujeto se compromete en la actividad para experimentar sensaciones asociadas a sus propios sentidos).

Desde la perspectiva de la teoría de la Integración Organísmica, tienen una gran importancia los procesos de interiorización y de integración. Las personas interiorizan las distintas regulaciones y las asimilan a través del ego, experimentando mayor autonomía en la acción. Los distintos tipos de regulación constituyen el llamado PLOC (Perceived Locus Of Causality), o locus percibido de causalidad, relacionado con el locus de control (externo o interno). Es normalmente medido a través 
de los diferentes tipos de razones (los motivos) para comprometerse en la conducta social (Deci \& Ryan, 2012; Hagger \& Armitage, 2004). Es un indicador de los distintos niveles de autonomía en la conducta.

En el ámbito deportivo, la variable ‘motivación' ha sido ampliamente estudiada en deportistas convencionales, pero en el contexto específico, como es el de los deportistas con discapacidad visual se dispone de poca información, por consiguiente, se retoman algunas investigaciones aplicadas al contexto del deporte, como lo son, la investigación de Moreno y Martínez (2006), en la que expresan que la Teoría de la Autodeterminación (Deci \& Ryan, 2012) constituye un modelo explicativo de la motivación humana que ha sido aplicado a diversos ámbitos, entre ellos, la actividad físico-deportiva, evidenciando que dicha teoría determina en qué medida las personas se involucran o no libremente en la realización de sus actividades, teniendo en cuenta una serie de mecanismos psicológicos reguladores de la conducta, y buscando en la medida de lo posible una mayor orientación hacia la motivación autodeterminada.

Además, en otra investigación realizada por Moreno, Cervelló y González (2007) trataron de establecer relaciones entre los elementos que definen la teoría de las Metas de Logro de Nicholls y la teoría de la Autodeterminación de Deci y Ryan, así como analizar las diferencias existentes en función de una serie de variables demográficas. Los resultados revelan que los deportistas con un alto índice de autodeterminación manifiestan una mayor orientación hacia la tarea y distinguen en mayor medida un clima tarea que los deportistas con un bajo índice, que son los que poseen una mayor orientación al ego y una mayor percepción de un clima ego. Además, los hombres revelan una mayor orientación al ego y perciben un clima motivacional más implicante al ego que las mujeres, que perciben un clima motivacional más implicante a la tarea y tienen un mayor índice de autodeterminación. También hallaron que los deportistas más jóvenes perciben un mayor clima tarea, y que un mayor tiempo de práctica se relaciona con un mayor índice de autodeterminación. Los deportistas de deportes co- 
lectivos tienen una mayor orientación al ego y una mayor percepción de un clima ego, mientras que en los deportes individuales es mayor la orientación a la tarea y el índice de autodeterminación.

En el ámbito de la Educación Física, Moreno, Conte, Hellín, Hellín, Vera y Cervelló (2008), realizaron una investigación con el fin de comprobar el poder de predicción de las estrategias utilizadas por el profesorado para mantener la disciplina y la orientación de meta disposicional sobre la motivación autodeterminada en estudiantes adolescentes de educación física, y la relación entre las mismas. Para ello, emplearon una muestra de 736 estudiantes correspondiente a los niveles educativos de Educación Secundaria Obligatoria y Bachillerato a los que se les pasaron las escalas de Estrategias para Mantener la Disciplina en Clase de educación física (SSDS), la Escala de Orientación Motivacional (POSQ) y la Escala de Motivación Deportiva (SMS). Los resultados mostraron la importancia de dirigir al alumnado a través de estrategias de disciplina de preocupación y responsabilidad para conseguir una orientación a la tarea y como consecuencia una motivación autodeterminada. Además, las estrategias de preocupación/responsabilidad del profesorado y la orientación al ego aparecieron como factores clave en el desarrollo de la motivación autodeterminada.

Por su parte, al estudiar el deporte en personas con discapacidad, las investigaciones son realmente escasas, aunque se destaca la realizada por Gutiérrez y Caus (2006), donde se retoma la teoría de las metas de logro, para conocer los motivos por los que se practica actividad física y deporte, para seguidamente analizar las posibles diferencias motivacionales de una muestra de 80 personas con discapacidad funcional y otras 80 sin ningún tipo de discapacidad practicantes de actividades físico-deportivas. Los resultados obtenidos señalan que los deportistas con discapacidad están más orientados al ego que los deportistas sin discapacidad, y obtienen puntuaciones más elevadas en los factores de integración social y afectividad social, como motivos para su práctica físico-deportiva.

Siguiendo con las investigaciones realizadas, Alonso y Pérez (2011) analizaron la motivación hacia la práctica deportiva en 100 deportis- 
tas habituales e igualmente evaluaron los motivos asociados para el mantenimiento de dicha práctica. Los resultados indicaron que la motivación hacia la práctica no era diferente según los factores estudiados de edad, fumador/no fumador, frecuencia de práctica, sin embargo, si influía la discapacidad en la práctica y el grado de discapacidad. Sólo el género y el tiempo con discapacidad mostraron diferencias significativas. Concretamente las personas con discapacidad física revelaron un alto nivel de motivación autodeterminada hacia la actividad físico-deportiva, asimismo mostraron un nivel más elevado que los practicantes sin discapacidad.

Por otra parte, un estudio realizado por García (2013) tuvo como propósito analizar los beneficios saludables que aporta el deporte para personas con discapacidad, teniendo en cuenta la motivación y su relación con el nivel de actividad física y su percepción de salud, en una muestra de cinco deportistas de diferentes modalidades deportivas. Los resultados sobre el nivel de actividad física deja entrever que algunos deportistas ocupan el tiempo de ocio en realizar algún tipo de actividad deportiva, con respecto a la motivación y a pesar de la heterogeneidad de la muestra, los deportistas obtuvieron valores similares, subrayando que realizan actividad deportiva sin esperar ningún elogio, sino, que la practican por el placer que experimentan, también indican que practican deporte aunque no sea agradable, ya que la práctica les permite tener beneficios físicos y psicológicos.

Y, por último, Torralba, Braz y Rubio (2014) examinaron la motivación hacia la práctica deportiva de 134 personas con discapacidad que forman parte del deporte federado. Los resultados indican que no existen diferencias ni entre ciegos/discapacitados visuales y discapacitados físico motriz, aunque cabe destacar la importancia dada a los factores de condición física y salud, tales como la práctica de deporte, la mejora del nivel, el competir, el sentirse bien o divertirse, que están muy por encima de ser popular, por la influencia de los entrenadores o por satisfacer a los padres.

Para el análisis de la motivación el instrumento más utilizado ha sido la Escala de Motivación Deportiva (SMS, Pelletier et al., 1995). Un 
análisis de las propiedades psicométricas des este instrumento fue realizado por Núñez, Martin-Albo \& Navarro-Izquierdo (2007), con una muestra formada por 496 deportistas, 314 hombres y 182 mujeres. El análisis factorial confirmatorio mostró una estructura de siete factores correlacionados al tiempo que mostraba niveles satisfactorios de consistencia interna y finalmente, y se han obtenido diferencias de género significativas. Estos resultados apoyan parcialmente el uso de la SMS para la evaluación de la motivación en el contexto deportivo.

Paralelamente, Balaguer, Castillo y Duda (2007), también analizaron las propiedades psicométricas del SMS, en esta ocasión con una muestra de 301 deportistas españoles. De los modelos hipotetizados el modelo de siete factores fue el que presentó un mejor ajuste de los datos, mostrando todas sus escalas una aceptable consistencia interna. Las correlaciones entre las subescalas ofrecieron evidencia para el continuo de la autodeterminación y la validez de constructo de la escala. En este estudio también emergieron diferencias por género en las regulaciones de la motivación deportiva indicando que las mujeres deportistas informaban menores niveles de regulación externa y de desmotivación que los hombres deportistas.

\section{Método}

El enfoque presentado en este estudio es cuantitativo, en el cual se realizó una medición de las diferentes variables que muestren la motivación autodeterminada. El estudio presenta resultados de frecuencias, sobre cómo se encontraron las variables. Es de tipo transversal, dando cuenta de la situación en un lugar y tiempo determinado.

\section{Muestra}

Se ha utilizado una muestra de 109 deportistas (90 hombres y 19 mujeres) con discapacidad visual. El rango de edad de la muestra va desde los 18 hasta los 35 años. La edad media es de 26.05 años y presenta una desviación típica de 5.41. El 64.2\% de la muestra tiene discapacidad total y el $35.8 \%$, parcial. 


\section{Instrumentos}

Se utilizó la Escala de Motivación Deportiva (SMS-28; Pelletier et al ., 1995) en la versión española de Balaguer, Castillo y Duda (2007). La escala está compuesta por 28 ítems y consta de siete subescalas de cuatro ítems cada una que miden los tres tipos de motivación intrínseca (MI orientada al conocimiento, MI orientada al logro y MI orientada a las experiencias estimulantes), los tres tipos de motivación externa o extrínseca (regulación externa, regulación introyectada y regulación identificada) y la amotivación. Cada uno de los ítems son respuestas a la pregunta: ¿Por qué prácticas tu deporte?, y se puntúan de acuerdo con una escala tipo Likert de siete puntos desde 1 ('No se corresponde en absoluto') hasta 7 ('Se corresponde totalmente').

Además, se incluyó un cuestionario sociodemográfico para recoger información sobre el género, la edad, tipo de deficiencia y años de práctica.

\section{Procedimiento}

A los deportistas en situación de discapacidad visual la investigadora les leyó las 28 preguntas que corresponden al Cuestionario de Motivación Deportiva (SMS-28) y la escala para calificarlo, este fue cumplimentado en los escenarios deportivos dispuestos para los IX Juegos Nacionales para Deportistas con Discapacidad Visual. Previamente, se dieron las directrices para que la escala y el cuestionario sociodemográfico se pudieran cumplimentar adecuadamente. En todo momento se señaló la voluntariedad, la finalidad y el anonimato de los resultados de la investigación.

\section{Análisis estadístico}

Se llevó a cabo un análisis descriptivo y un análisis inferencial de los datos. En el primero, se examinaron las medias y desviaciones típicas; en el segundo, se compararon las medias obtenidas por los deportistas en la Escala de Motivación Deportiva a través de la prueba $t$ de Student, para comprobar si existían diferencias significativas. Por último, 
se efectuó un análisis correlacional de Pearson para conocer si existía relación, entre la escala y las variables sociodemográficas. Los datos se analizaron con el paquete estadístico SPSS 21.0 para Windows.

\section{Resultados}

\section{Consistencia interna y fiabilidad}

Se calculó la consistencia interna de la Escala de Motivación Deportiva, la alfa de Cronbach fue de .838 , superior a la obtenida por Vallerand et al . (1989), que era de .80. Para cada una de las subescalas, también se halló la consistencia interna, con los siguientes resultados: .49 para la desmotivación; .73 para la regulación externa; .71 para la regulación introyectada; .65 para la regulación identificada; y .79 para la MI orientada al conocimiento; .75 para la MI orientada al logro; .81 para la MI orientada a las experiencias estimulantes.

\section{Análisis descriptivo y diferencias según género, edad y tipo de discapacidad}

Para el análisis de las diferencias de medias en la escala de motivación, en función del género, la edad y el tipo de discapacidad, se utilizó la t de Student teniendo en cuenta el test de Levene para estimar la igualdad de las varianzas.

Los resultados revelan que existen diferencias significativas entre las medias obtenidas por hombres y mujeres en las subescalas de Regulación Introyectada y de Regulación Externa, siendo mayor en los hombres que en las mujeres (véase Tabla 1).

Tabla 1. Diferencias de medias y desviación típica en las variables de motivación y la variable género

\begin{tabular}{|lcccc|}
\hline & $\begin{array}{c}\text { HOMBRE } \\
(\mathbf{n}=\mathbf{9 0})\end{array}$ & $\begin{array}{c}\text { MUJER } \\
(\mathbf{n}=\mathbf{1 9})\end{array}$ & & \\
\hline & M y DT & M y DT & t & p \\
\hline Regulación Introyectada & $21.83(5.05)$ & $18.26(7.94)$ & 2.506 & $.014^{*}$ \\
\hline
\end{tabular}




\begin{tabular}{|lllll|}
\hline Regulación Identificada & $22.63(4.80)$ & $20.89(5.26)$ & 1.327 & .197 \\
\hline Regulación Externa & $16.67(6.40)$ & $12.42(7.41)$ & 2.561 & $.012^{*}$ \\
\hline Motivación Intrínseca Logro & $25.21(3.87)$ & $24.84(5.66)$ & .345 & .731 \\
\hline Motivación Intrínseca Conocimiento & $24.56(4.58)$ & $24.42(6.39)$ & .117 & .907 \\
\hline Motivación Intrínseca Experiencia & $25.75(3.51)$ & $25.52(5.53)$ & .231 & .817 \\
\hline Amotivación & $6.25(3.17)$ & $6.00(2.70)$ & .362 & .720 \\
\hline
\end{tabular}

Nota: $* p<.05 ; * * p<.01 ; * * * p<.001$

\section{Fuente: Autores}

Los resultados de la comparación de medias con muestras independientes entre el grupo con edades comprendidas entre los 18 y 26 años (jóvenes) y entre 27 y 35 años (adultos) (Tabla 2), la comparación de medias con respecto al tipo de discapacidad, total o parcial (Tabla 3), y la comparación de medias entre el grupo con años de práctica comprendidas entre los 1 y 10 años y los 11 y 20 años (Tabla 4) no arrojaron diferencias significativas entre las distintas subdimensiones.

Tabla 2. Diferencias de medias y desviación típica en las variables de motivación y la variable edad

\begin{tabular}{|lcccc|}
\hline & $\begin{array}{l}\text { JÓVENES } \\
(\boldsymbol{n}=\mathbf{5 7})\end{array}$ & $\begin{array}{l}\text { ADULTOS } \\
(\boldsymbol{n}=\mathbf{5 2})\end{array}$ & & \\
\hline & M y DT & M y DT & $\mathrm{t}$ & $\mathrm{p}$ \\
\hline Regulación Introyectada & $21.50(5.83)$ & $20.88(5.75)$ & .562 & .575 \\
\hline Regulación Identificada & $22.31(4.79)$ & $22.34(5.07)$ & -.032 & .974 \\
\hline Regulación Externa & $15.61(6.79)$ & $16.2(6.75)$ & -.519 & .605 \\
\hline Motivación Intrínseca Logro & $24.77(4.01)$ & $25.55(4.43)$ & -.972 & .333 \\
\hline Motivación Intrínseca Conocimiento & $24.24(4.79)$ & $24.86(5.07)$ & -.656 & .514 \\
\hline Motivación Intrínseca Experiencia & $25.91(3.32)$ & $25.50(4.47)$ & .549 & .584 \\
\hline Amotivación & $6.05(2.60)$ & $6.38(3.57)$ & -.558 & .578 \\
\hline
\end{tabular}

Fuente: Autores 
Tabla 3. Diferencias de medias y desviación típica en las variables de motivación y la variable tipo de discapacidad

\begin{tabular}{|lllll|}
\hline & $\begin{array}{l}\text { TOTAL } \\
(\boldsymbol{n}=\mathbf{7 0})\end{array}$ & $\begin{array}{l}\text { PARCIAL } \\
(\boldsymbol{n}=\mathbf{3 9})\end{array}$ & \\
\hline & M y DT & M y DT & $\mathrm{t}$ & $\mathrm{p}$ \\
\hline Regulación Introyectada & $20.74(5.88)$ & $22.05(5.56)$ & -1.135 & .259 \\
\hline Regulación Identificada & $22.27(5.22)$ & $22.43(4.35)$ & -.167 & .868 \\
\hline Regulación Externa & $15.27(6.92)$ & $17.12(6.33)$ & -1.382 & .170 \\
\hline Motivación Intrínseca Logro & $24.95(4.64)$ & $25.48(3.22)$ & -.627 & .532 \\
\hline Motivación Intrínseca Conocimiento & $24.22(5.25)$ & $25.10(4.24)$ & -.889 & .376 \\
\hline Motivación Intrínseca Experiencia & $25.67(4.20)$ & $25.79(3.35)$ & -.157 & .875 \\
\hline Amotivación & $6.5(3.25)$ & $5.69(2.73)$ & 1.312 & .192 \\
\hline
\end{tabular}

Fuente: Autores

Tabla 4. Diferencias de medias y desviación típica en las variables de motivación y la variable años de práctica del deporte

\begin{tabular}{|c|c|c|c|c|}
\hline & $1 \mathrm{~A} 10 \mathrm{AÑOS}$ & 11 A 20 AÑOS & & \\
\hline & $(n=96)$ & $(n=13)$ & & \\
\hline & M y DT & M y DT & $\mathrm{t}$ & $\mathrm{p}$ \\
\hline Regulación Introyectada & $21.15(5.92)$ & $21.69(4.76)$ & -.319 & .751 \\
\hline Regulación Identificada & $22.22(4.87)$ & $23.07(5.25)$ & -.583 & .561 \\
\hline Regulación Externa & $15.58(6.83)$ & $18.53(5.69)$ & -1.489 & .139 \\
\hline Motivación Intrínseca Logro & $25.11(4.25)$ & $25.38(4.11)$ & -.221 & .828 \\
\hline $\begin{array}{l}\text { Motivación Intrínseca Cono- } \\
\text { cimiento }\end{array}$ & $24.56(4.86)$ & $24.38(5.50)$ & .122 & .903 \\
\hline $\begin{array}{l}\text { Motivación Intrínseca Expe- } \\
\text { riencia }\end{array}$ & $25.70(3.87)$ & $25.76(4.30)$ & -.053 & .958 \\
\hline Amotivación & $6.14(2.86)$ & $6.69(4.53)$ & -.596 & .552 \\
\hline
\end{tabular}

Fuente: Autores 


\section{Correlaciones entre la variable motivación y las variables sociodemográ- ficas}

El análisis de las correlaciones expone que tanto se relacionan las variables que han sido determinadas para esta investigación, en este caso, dar cuenta, de la dependencia o no, entre la variable motivación con la edad y los años de práctica de los deportistas.

En lo que respecta a las variables sociodemográficas sólo se observa una correlación estadísticamente significativa y positiva, entre los años de práctica del deporte y la Regulación externa, de $r=.216$, mientras que no aparecen correlaciones significativas entre la edad y las distintas subdimensiones de la motivación (Tabla 5).

La correlación entre las subescalas de la ME se encuentran que son estadísticamente significativas y positivas entre la Regulación Introyectada con Regulación Identificada $(r=.585)$, y Regulación Introyectada con Regulación Externa $(r=.475)$, de la misma manera la Regulación Identificada correlaciona con la Regulación Externa $(r=.578)$

También, se evidencian correlaciones significativas y positivas entre la MI al Logro con MI al Conocimiento $(r=.804)$; MI al Logro con MI a Experiencias Estimulantes, de $r=.801 ;$ y la MI al Conocimiento con la MI a Experiencias Estimulantes, de $r=.642$.

En lo que respecta a la correlación entre las subescalas de la ME y la MI estas son fuertes y positivas, es así como la Regulación Introyectada correlaciona con la MI al Logro con $r=.499$, con la MI al Conocimiento $(r=.487)$ y con la MI a Experiencias Estimulantes $(r=.424)$.

Por su parte la Regulación Identificada correlaciona con la MI al Logro $(r=.644)$, MI al Conocimiento $(r=.688)$ y con la MI a Experiencias Estimulantes $(r=.545)$; en cuanto a la Regulación Externa esta correlaciona de forma significativa con las tres MI, al Logro con $r=.407$, al Conocimiento con $r=.506$, y a Experiencias Estimulantes $\operatorname{con} r=.353$.

$\mathrm{Y}$, por último, las subescalas de la MI correlaciona entre sí, la MI al Logro con MI al Conocimiento $(r=.804)$ y con Experiencias Esti- 
mulantes ( $r=.801)$; y la MI al Conocimiento correlaciona con la MI a Experiencias Estimulantes $(r=.642)$.

Tabla 5. Correlación de Pearson entre la escala de motivación y la edad

\begin{tabular}{|c|c|c|c|c|c|c|c|c|c|}
\hline & 1 & 2 & 3 & 4 & 5 & 6 & 7 & 8 & 9 \\
\hline 1. Edad & & $.278^{* *}$ & -.038 & .021 & .054 & .160 & .109 & -.001 & .143 \\
\hline 2. Años de Práctica & & & .125 & .076 & $.216^{*}$ & .044 & .029 & .059 & .155 \\
\hline 3. Regulación Introyectada & & & & $.585^{* *}$ & $.475^{* *}$ & $.499^{* *}$ & $.487^{* *}$ & $.424^{* *}$ & .151 \\
\hline 4. Regulación Identificada & & & & & $.578^{* *}$ & $.644^{* *}$ & $.668^{* *}$ & $.545^{* *}$ & .148 \\
\hline 5. Regulación Externa & & & & & & $.407^{* *}$ & $.506^{* *}$ & $.353^{* *}$ & .091 \\
\hline $\begin{array}{l}\text { 6. Motivación Intrínseca } \\
\text { Logro }\end{array}$ & & & & & & & $.804^{* *}$ & $.801^{* *}$ & -.001 \\
\hline $\begin{array}{l}\text { 7. Motivación Intrínseca } \\
\text { Conocimiento }\end{array}$ & & & & & & & & $.642^{* *}$ & .082 \\
\hline $\begin{array}{l}\text { 8. Motivación Intrínseca } \\
\text { Experiencia }\end{array}$ & & & & & & & & & -.069 \\
\hline 9. Amotivación & & & & & & & & & \\
\hline
\end{tabular}

Nota: ${ }^{*} p<.05 ;{ }^{* *} p<.01 ;{ }^{* * *} p<.001$

Fuente: Autores

\section{Discusión y Conclusiones}

Retomando a Deci \& Ryan $(1985,2000)$ con la Teoría de la Autodeterminación (TAD) explican que la conducta de un deportista puede estar determinada intrínsecamente, extrínsecamente o no estar motivada (amotivación), donde cada una de estas difiere entre resultados bajos (amotivación) a muy altos (motivación intrínseca), estas últimas, constituyen el ideal autodeterminado (Deci, 1975), es decir, que a mayor grado a autodeterminación mayor desarrollo del bienestar (Deci \& Ryan, 1985, 2000).

Por ende y de acuerdo a la información anterior, se puede observar que existen diferencias significativas determinadas con respecto al género en cuanto al nivel de motivación. Estos resultados coinciden 
con los encontrados por Miller (2000), Brière, Vallerand, Blais \& Pelletier (1995) y Núñez, Martín-Albo, Navarro \& González (2006) donde el género masculino puntuaba más alto en regulación externa que el femenino. De la misma manera, el estudio realizado por Pelletier, Fortier, Vallerand, Tuson, Brikre \& Blais (1995) destacan que las mujeres puntuaban más alto en motivación intrínseca y más bajo en motivación extrínseca y desmotivación que los hombres. Asimismo, (Chantal, Guay, Dobreva y Vallerand, 1996), revelan en su estudio que las mujeres puntúan una motivación intrínseca más alta y una motivación extrínseca más baja que los hombres. Igualmente, Balaguer, Castillo y Duda (2007) en la investigación que llevaron a cabo con sobre las "Propiedades psicométricas de la escala de motivación deportiva en deportistas españoles” señalan que los hombres y las mujeres deportistas difirieren significativamente en la motivación extrínseca, concretamente los hombres deportistas señalaron mayor regulación externa que las mujeres deportistas. Por ende, se puede concluir en cuanto a la variable género, que los hombres muestran un perfil menos autodeterminado que las mujeres.

Observando los resultados obtenidos con la aplicación del SMS-28 (Escala de Motivación Deportiva de Pelletier et al ., 1995; Balaguer, Castillo y Duda, 2003 y 2007) por la media de cada regulación, se puede comentar que los resultados de la Motivación Extrínseca $(M=59.48)$, donde cada una de sus subescalas evidencia valores dentro de la media, menos el de la Regulación Externa que tienen hacer bajo (Regulación Introyectada $M=21.21$; Regulación Identificada $M=22.33$, y la Regulación Externa $M=15.94$ ) lo cual permite concluir que los deportistas con discapacidad visual la realizarán aunque no fuera agradable, ya que ellos son conscientes que la práctica deportiva les reporta beneficios físicos y psicológicos. Por su parte, la Motivación Intrínseca $(M=$ 75.40), evidencia una mayor puntuación refiriendo que la Motivación Intrínseca a la Experiencia con una puntuación alta $(M=25 \cdot 72)$, la Motivación Intrínseca al Logro también es alta $(M=25.15)$ y por último, la Motivación Intrínseca al Conocimiento $(M=24.54)$, lo cual permite concluir, que los deportistas con discapacidad visual reflejan el sentimiento que obtienen de la experiencia física, de la sensación de que tiene el don para realizar determinada tarea (Cox, 2009), de la misma 
manera Vallerand et al . $(1989,1993)$ resaltan que el sujeto se compromete en la actividad para experimentar sensaciones asociadas a sus propios sentidos. Para finalizar, se observa los resultados de Amotivación, el cual es bajo $(M=6.21)$, es decir que ninguno de los deportistas tiene falta de motivación.

Por su parte, en lo que respecta al análisis de correlación, se encontró que la Motivación Extrínseca (ME) correlaciona fuerte y positivamente con la Motivación Intrínseca (MI) en cada una de sus subescalas, por lo que se sustenta el principio de Deci \& Ryan (1985) referido a la presencia de un continuo de autodeterminación, desde la Amotivación a la MI, en el que las subescalas inmediatos muestran correlaciones más altas que aquellas opuestas en el continuo; se destaca la correlación más alta y significativa entre la Regulación Identificada y la Motivación al Conocimiento $(r=.668)$, mientras que la más baja es la de Regulación Externa con Motivación Intrínseca a la Experiencia $(r=.353)$, lo cual evidencia lo referido por los autores anteriormente nombrados.

En lo que respecta a las correlaciones de las variables sociodemográficas con el continuo de la autodeterminación, se observa que correlacionan años de práctica con la Regulación Externa, la cual de acuerdo con Deci \& Ryan (2000), los deportistas practican deporte para satisfacer una demanda externa o por los beneficios que ello pueda traer, por lo que tienen un locus de control externo, es decir, que muchos deportistas pueden practicar deporte porque "los demás me dicen que debo hacerlo", "los amigos, la familia, mi pareja, etcétera me presionan para que haga ejercicio", "el entrenador me dice que debo mejorar mi marca en 5 segundos si quiero hacer algo esta temporada", etcétera. (Ryan \& Deci, 2000).

Para finalizar, se destaca la pertinencia del uso de la Escala de Motivación Deportiva (SMS-28) con la población de deportistas con discapacidad visual, ya que solventa y permite la elaboración de un perfil de puntos fuertes/débiles con respecto a la motivación. 


\section{Referencias}

Alonso, J. y Pérez, J. (2011, febrero). La motivación autodeterminada y disfrute en deportistas con discapacidad física: sugerencias para la inclusión. Póster presentando en la II Conferencia Nacional de Deporte Adaptado. Universidad de Valencia, Valencia, España. Recuperado de http://www.csd.gob.es/csd/ estaticos/dep-adp/conda2/posters/10-MOTIVACION-AUTODETERMINADA-EN-DEPORTISTAS-CON-DISCAPACIDAD.pdf

Bakker, F., Whiting, H. y Van der Brug, H. (1993). Psicología del deporte. Conceptos y Aplicaciones. Madrid: Morata.

Balaguer, I., Castillo, I. y Duda, J. L. (2007). Propiedades psicométricas de la Escala de Motivación Deportiva en deportistas españoles. Revista Mexicana de Psicología, 24(2), 197-207. Recuperado de https://selfdeterminationtheory.org/ SDT/documents/2007_BalaguerCastilloyDuda_RMP.pdf

Balaguer, I., Castillo, I., y Duda, J. L. (2003). La Escala de Motivación Deportiva: una nueva medida de la motivación intrínseca, motivación extrínseca y no-motivación. Análisis psicométrico de la versión española. En libro de resúmenes del II Congreso Internacional de Psicología Aplicada al Deporte (165). Madrid: Dykinson.

Bandura, A. (2001). Social cognitive theory: An Agentic Perspective. Annual Review of Psychology, 52, 1-26. Recuperado de https://www.uky.edu/ eushe2/Bandura/Bandura2001ARPr.pdf

Brière, N. M., Vallerand, R. J., Blais, M. R. y Pelletier, L. G. (1995). Développement et validation d'une mesure de motivation intrinsèque et d'amotivation en contexte sportif: l'Échelle de Motivation dans les Sports (EMS). International Journal of Sport Psychology, 26, 465-489. Recuperado de https:// www.researchgate.net/publication/232440657_Developpement_et_Validation_d'une_Mesure_de_Motivation_Intrinseque_Extrinseque_et_d'Amotivation_en_Contexte_Sportif_L'Echelle_de_Motivation_dans_les_Sports_ EMS_Development_and_validation_of_a_scale_on 
Carratalá, E. (2004). Análisis de la teoría de las metas de logro y de la autodeterminación en los planes de especialización deportiva de la Generalitat Valenciana. Tesis doctoral sin publicar, Universidad de Valencia, Valencia (España). Recuperado de http://www.tdx.cat/bitstream/handle/10803/10183/carratala. pdf? sequence $=1$

Cox, R. (2009). Psicología del Deporte: conceptos y sus aplicaciones (6ª Ed.). Madrid: Médica Panamericana.

Chantal, Y., Guay, F., Dobreva-Martinova, T. y Vallerand, R. J. (1996). Motivation and elite performance: An exploratory investigation with Bulgarian athletes. International Journal of Sport Psychology, 27, 173-182. Recuperado de https://selfdeterminationtheory.org/SDT/documents/1996_ChantalGuayDobrevaVallerand_IJSE.pdfpdf.pdf

Deci, E. L. (1975). Intrinsic motivation. Nueva York: Plenum

Deci, E. L. y Ryan, R. M. (1985). Intrinsic motivation and self-determination in human behavior. Nueva York: Plenum Press.

Deci, E. L. y Ryan, R. M. (2000). The "what" and "why" of goal pursuits: Human needs and the self-determination of behavior. Psychological Inquiry, 11, 227268. doi: 10.1207/S15327965PLI1104_01

Deci, E. L. y Ryan, R. M. (2002). Handbook of self-determination research. Rochester, NY: University of Rochester Press.

Deci, E. L. y Ryan, R. M. (2008). A self-determination theory approach to psychotherapy: The motivational basis for effective change. Canadian Psychology, 49(3), 186-193. doi: 10.1037/aoo12753

Deci, E. L. y Ryan, R. M. (2012). Motivation, personality and development within embedded social context: An Overview of self-determination theory. En R. M. Ryan (Ed.), The Oxford Handbook of Human Motivation. Oxford, UK: Oxford University Press.

Edmunds, J., Ntoumanis, N. y Duda, J. (2006). A Test of Self-Determination Theory 
in the Exercise Domain. Journal of Applied Social Psychology, 36(9), 22402265. doi: 10.1111/j.0021-9029.2006.00102.x

García-Calvo, T. (2004). La motivación y su importancia en el entrenamiento con jóvenes deportistas. Madrid: Comunidad Virtual del Deporte.

García-González, L. (2013). Beneficios de la actividad física y deportiva en personas con discapacidad: La opinión de los deportistas. Trabajo Fin de Grado sin publicar. Universidad Politécnica de Madrid, Madrid, España. Recuperado de http://oa.upm.es/20549/1/TFG_LAURA_GARCIA_GONZALEZ.pdf

Gutiérrez, M. y Caus, N. (2006). Análisis de los motivos para la participación en actividades físicas de personas con y sin discapacidad. Revista Internacional de Ciencias del Deporte, 2(2), 49-64. doi:10.5232/ricyde2006.00204

Hagger, M. S. y Armitage, C. J. (2004). The Influence of Perceived Loci of Control and Causality in the Theory of Planned Behavior in a Leisure-Time Exercise Context. Journal of Applied Biobehavioral Research, 9(1), 45-64. doi: 10.1111/j.1751-9861.2004.tbooog1.x

Miller, J. A. (2000). Intrinsic, extrinsic and amotivational difference in scholarship and non scholarship collegiate track and field athletes. Tesis de Máster no publicada. Springfield College, Springfield, Massachusetts, USA.

Moreno, J. A., Cervelló, E. y González-Cutre, D. (2007). Analizando la motivación en el deporte: un estudio a través de la teoría de la autodeterminación. Apuntes de Psicología, 25(1), 35-51. Recuperado de http://www.um.es/univefd/deporteiad.pdf

Moreno, J. A., Conte, L., Hellín, P., Hellín, G., Vera, J. A. y Cervelló, E. (2008). Predicción de la motivación autodeterminada según las estrategias para mantener la disciplina y la orientación motivacional en estudiantes adolescentes de educación física. Apuntes de Psicología, 26(3), 501-516. Recuperado de http:// www.apuntesdepsicologia.es/index.php/revista/article/view/169/171 
Moreno, J. A., y Martínez, A. (2006). Importancia de la Teoría de la Autodeterminación en la práctica físico-deportiva: Fundamentos e implicaciones prácticas. Cuadernos de Psicología del Deporte, 6(2), 39-54. Recuperado de http:// www.um.es/univefd/TAD.pdf

Núñez, J. L., Martín-Albo, J., Navarro, J. G. y González, V. M. (2006). Preliminary validation of a Spanish version of the sport motivation scale. Perceptual and Motor Skills, 102, 919-930. doi: 10.2466/PMS.102.3.919-930

Núñez, J. L., Martín, J. y Navarro, J. G. (2007). Propiedades psicométricas de la versión española de la Escala de Motivación Deportiva. Revista de Psicología del Deporte, 16(2), 211-223. Recuperado de http://www.redalyc.org/articulo. oa?id=235119266007

Pelletier, L. G., Fortier, M. S., Vallerand, R. J., Tuson, K. M., Briere, N. M. \& Blais, M. R. (1995). Toward a new measure of intrinsic motivation, extrinsic motivation, and amotivation in sports: The sport motivation scale (SMS). Journal of Sport and Exercise Psychology, 17, 35-53. Recuperado de http://selfdeterminationtheory.org/SDT/documents/1995_PelletierFortierVallerandTuson_JSEP.pdf

Ryan, R. M., y Deci, E. L. (2000). The darker and brighter sides of human existence: Basic psychological needs as a unifying concept. Psychological Inquiry, 11(4), 319-338. doi: 10.1207/S15327965PLI1104_03

Seligman, M. E. P. (1975). Helplessness. San Francisco, CA: W. H. Freeman.

Torralba, M., Braz, M. y Rubio, M. (2014). La motivación en el deporte adaptado. Psychology, Society, \& Education, 6(1), 27-40. Recuperado de http://www. psye.org/articulos/torralba.pdf?EsetProtoscanCtx $=8$ edo498

Vallerand, R. J. (2001). A hierarchical model of intrinsic and extrinsic motivation in sport and exercise. En G. C. Roberts (Ed.), Advances in motivation in sport and exercise (pp. 263-319). Champaign, IL: Human Kinetics.

Vallerand, R. J., Blais, M. R., Brière, N. M. y Pelletier, L. G. (1989). Construction et validation de l'Échelle de motivation en éducation (EME). Canadian Journal 
of Behavioural Science, 21, 323-349. Recuperado de http://selfdeterminationtheory.org/SDT/documents/1989_VallerandBlaisBrierePelletier_CJBSRCSC.pdf

Vallerand, R. J., Pelletier, L. G., Blais, M. R., Brière, N. M., Senécal, C. y Vallières, E. F. (1993). On the assessment of intrinsic, extrinsic and amotivation in education: evidence on the concurrent and construct validity of the Academic Motivation Scale. Educational and Psychological Measurement, 53, 159-172. doi: 10.1177/0013164493053001018

Villacorta, M., Koestner, R. y Lekes, N. (2003). Further Validation of the Motivation toward the Environment Scale. Environment and Behavior, 35(4), 486-505. doi: 10.1177/0013916503035004003

Weinberg, R. y Gould, D. (2010). Fundamentos de psicología de la actividad física y del deporte (4 ${ }^{\mathrm{a}}$. Edición). Madrid: Ed. Medica Panamericana. 